\title{
USO DO PLANEJAMENTO EXPERIMENTAL PARA O DESENVOLVIMENTO DE TINTAS E ADESIVOS ANTICORROSIVOS
}

\author{
Danielly Vieira de Lucena ' \\ Danyllo Vieira de Lucena ${ }^{2}$ \\ Ariosvaldo Alves Barbosa Sobrinho ${ }^{3}$
}

\section{Resumo}

O objetivo deste trabalho é estudar a influência do teor de argila e do número de camadas "ativas" nos parâmetros de grau de enferrujamento e no grau de formação de bolhas, utilizando um planejamento fatorial do tipo $2^{2}$ com três experimentos no ponto central. Para isto, foram obtidos nanocompósitos polímero-argila, usando montmorilonita (MMT), como fase inorgânica, e polimetacrilato de metila (PMMA), como fase orgânica, para uso como revestimentos e adesivos (tintas e vernizes) com alta resistência à corrosão, para serem aplicados sobre estruturas metálicas utilizadas na indústria petrolífera. Também foram realizadas medidas de espessura e de aderência dos filmes para comprovar a eficácia do revestimento produzido. A partir da análise de superfícies de respostas observa-se que as melhores características anticorrosivas são observadas para os revestimentos acrílicos aditivados com $5 \%$ de argila organofílica e três camadas "ativas". Esse comportamento é esperado devido ao maior reforço estabelecido pela elevada concentração dos componentes formadores do revestimento.

Palavras-chave: Planejamento fatorial; Corrosão; Revestimentos.

\section{USE OF EXPERIMENTAL PLAN TO DEVELOPMENT OF PAINTS AND STICKERS ANTICORROSIVES}

\begin{abstract}
The aim of this work is to study the influence of clay content and the number of layers "active" in the musical parameters and the degree of rust bubbles, using a experimental plan $2^{2}$ type with three experiments in the central point. For this, polymer-clay nanocomposites obtained using montmorillonite (MMT), as the inorganic phase, and poly(methyl methacrylate) (PMMA), as organic phase, for use as adhesives and coatings (paints and varnishes) with high resistance to corrosion, to be applied on metal structures used in the petroleum industry. Measures of thickness and adhesion of films to demonstrate the effectiveness of the coating produced were also conducted. From the analysis of response surfaces show that the best corrosion characteristics are observed for acrylic coatings fortified with $5 \%$ organoclay layers and three "active". This behavior is expected on account of the further enhancement provided by the high concentration of the components forming coating.
\end{abstract}

Key words: Experimental plan; Corrosion; Coating.

\footnotetext{
'Doutoranda em Ciências e Engenharia de Materiais, Universidade Federal de Campina Grande - UFCG, Rua Basílio Araújo, 886, Catolé, Cep 58410-200, Campina Grande, PB, Brasil. E-mail: daniellymateriais@yahoo.com.br

${ }^{2}$ Graduando em Engenharia Ambiental e Sanitária, Universidade Estadual da Paraíba - UEPB, Rua Basílio Araújo, 886, Catolé, Cep 584I0-200, Campina Grande, PB, Brasil. E-mail: danyllo_vieira@gmail.com

${ }^{3}$ Professor doutor da Unidade Acadêmica de Engenharia de Materiais, Universidade Federal de Campina Grande - UFCG, Rua Aprígio Veloso, 882 ,

Universitário, Campina Grande, PB, Brasil. E-mail: ariosvaldo@dema.ufcg.edu.br
} 


\section{INTRODUÇÃO}

Uma das preocupações atuais dos diversos ramos industriais é o problema de deterioração de estruturas metálicas devido à ação corrosiva de vários meios a que se acham submetidas. Assim, a busca por novos métodos de prevenção e, até mesmo desenvolvimento de novos materiais com características anticorrosivas, vem sendo amplamente estudados e discutidos na indústria petrolífera. Dentre as diversas metodologias aplicadas, destaca-se o uso de revestimentos orgânicos comercializados mundialmente e tintas à base de polímeros. ${ }^{(1)}$

Os processos reacionais que geram a corrosão são geralmente espontâneos e transformam, constantemente, as características físicas e químicas de materiais metálicos e não-metálicos, interferindo, em consequência, nas suas estruturas, durabilidade e no seu desempenho. $O$ conhecimento exato dos princípios básicos desses processos é imprescindível para que se estabeleçam considerações qualitativas e quantitativas sobre a cinética de corrosão. Dentre as muitas maneiras de definição de corrosão, pode-se afirmar que: "[...] a corrosão é a deterioração espontânea de um material, metálico ou não metálico, por ação química ou eletroquímica do meio ambiente, aliada, ou não, a esforços mecânicos".(2,3) Uma vez que todas as estruturas metálicas estão suscetíveis a esse processo deteriorativo e que o uso das técnicas de revestimentos protetores vem sendo continuamente estudado, este trabalho ressalta a importância do estudo deste tema, principalmente para a indústria de petróleo.(4)

Um dos principais meios causadores de corrosão em instalações industriais são as salmouras. Um exemplo significativo pode ser dado na indústria de óleos, onde a corrosão em oleodutos é desencadeada por elevados teores de salinidade, necessários para a produção de óleos. ${ }^{(3)} \mathrm{A}$ literatura relata um grande número pesquisas que têm sido desenvolvidas no sentido de reduzir os efeitos da corrosão. $O$ uso de inibidores de corrosão caracteriza-se em um dos métodos de grande interesse, já que funcionam como películas protetoras que interferem na ação eletroquímica. ${ }^{(3)}$ Como inibidores de corrosão, moléculas orgânicas fortemente polares são alvo de interesse na indústria de petróleo, já que promovem a formação de um filme protetor na interface metal-meio corrosivo. . $^{(5-8)}$

Os revestimentos orgânicos, em geral, podem ser obtidos em forma de pó, em solução orgânica ou em dispersão, que pode ser aquosa ou não-aquosa Este último tipo dá origem a organossóis e plastissóis. ${ }^{(9)}$ Os revestimentos, ainda, podem ser formulados na forma de materiais nanoestruturados poliméricos, os quais fazem parte de uma classe de materiais híbridos compostos por uma matriz polimérica orgânica, na qual estão inseridas partículas inorgânicas em escala nanométrica. ${ }^{(10)}$
O desenvolvimento de materiais nanoestruturados polímero/argila é uma das maiores evoluções na tecnologia dos polímeros, pois eles oferecem um grande potencial de aplicação e diversificação dos materiais poliméricos convencionais. ${ }^{(8)}$ Essas nanoestruturas consistem de uma nova classe de materiais, com propriedades elevadas, empregando níveis muito baixos de aditivos argilominerais organofílicos, quando comparados com os materiais compósitos convencionais. (II)

As argilas organofílicas nanométricas, com elevada razão de aspecto, no caso dos materiais nanoestruturados, exercem um efeito de barreira mais acentuado, pois são impermeáveis, e faz com que o penetrante precise cada vez mais desviar dos obstáculos (partículas nanométricas), tornando a difusão um processo mais lento e difícil, como mostra a Figura I. (12)

Recentemente, as soluções encontradas para redução das perdas causadas pela corrosão das estruturas metálicas da indústria petrolífera tem sido, por exemplo, a substituição de tais estruturas metálicas por polímeros de engenharia de alto desempenho, assim como o desenvolvimento e aplicação de revestimentos (tintas, adesivos e vernizes) de alta resistência à corrosão para aplicação sobre estruturas metálicas.

A utilização do método de planejamento fatorial em estudos que abrangem variáveis independentes, como é o caso do desenvolvimento de revestimentos anticorrosivos, é importante, pois torna possível o planejamento e a realização de maneira organizada de uma quantidade mínima necessária de experimentos, economizando tempo e recursos financeiros. ${ }^{(13)}$ Deste modo, a utilização da metodologia de planejamento experimental é indispensável para que se possa atingir o objetivo deste trabalho que é estudar a influência do teor de argila e do número de camadas "ativas" nos parâmetros de grau de enferrujamento e no grau de formação de bolhas.

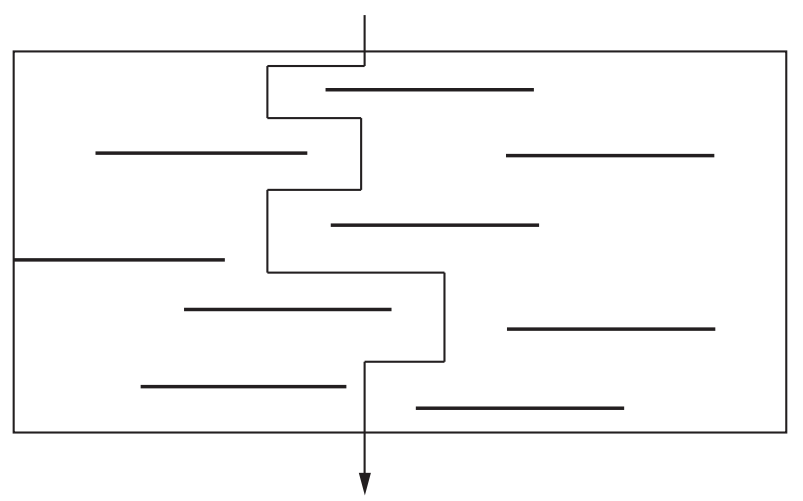

Figura I. Ilustração do caminho difusional percorrido por um penetrante através dos materiais nanoestruturados. 


\section{MATERIAIS}

\section{I Corpos de Prova}

Para este estudo foram utilizados corpos de prova em aço 1020, para aplicação no ensaio de imersão em solução de $\mathrm{NaCl}$.

\subsection{Revestimento}

Para preparação do filme anticorrosivo foram utilizados argila bentonítica Brasgel PA da cidade de Boa Vista/PB organofilizada com sal quaternário de amônio (cloreto de alquil dimetil benzil amônio), conhecido como DODIGEN I6I I da Chemco e polimetacrilato de metila como polímero acrílico.

Foram também selecionadas, para fins comparativos, três amostras de revestimentos anticorrosivos comerciais, disponíveis no mercado internacional, denominados de CORRPASSIV 4900, CORRPASSIV 4905 e CORPASSIV 4934, todos da marca Ormecon. As composições dessas amostras não foram divulgadas por pedido de sigilo por parte da empresa.

\section{METODOLOGIA}

\section{I Organofilização da Argila}

Inicialmente. foi realizado o tratamento das amostras de argilas montmoriloníticas com o sal quaternário de amônio denominado cloreto de alquil dimetil benzil amônio $\left(\mathrm{C}_{12} \mathrm{H}_{24} \mathrm{~N}^{+}\left(\mathrm{CH}_{3}\right)_{2} \mathrm{CH}_{2} \mathrm{C}_{6} \mathrm{H}_{5} \mathrm{Cl}\right)$, mais conhecido como Dodigen I6II, Chemco.

Para o tratamento de organofilização foram preparadas dispersões contendo $768 \mathrm{~mL}$ de água destilada e $32 \mathrm{~g}$ de argila. A argila foi adicionada com agitação mecânica concomitante (Figura 2) e, após a adição de toda argila, a agitação foi mantida por 20 minutos. Em seguida, foi adicionada uma solução contendo $20 \mathrm{~mL}$ de água destilada e $20,4 \mathrm{~g}$ do sal quaternário de amônio. A agitação foi mantida durante 20 minutos. Após 24 horas foi realizada a lavagem das partículas foi feita com $2.000 \mathrm{~mL}$ de água destilada, sendo depois secas em estufa à temperatura de $60^{\circ} \mathrm{C}$, por um período de 48 horas. Em seguida, foram passadas em peneira $A B N T n^{\circ} 200(D=0,074 \mathrm{~mm})$ para serem posteriormente caracterizadas. Este tratamento da argila vem facilitar sua atuação na estrutura do material nanoestruturado, levando a uma melhor intercalação da argila no polímero, além de melhorar suas características de adesão.

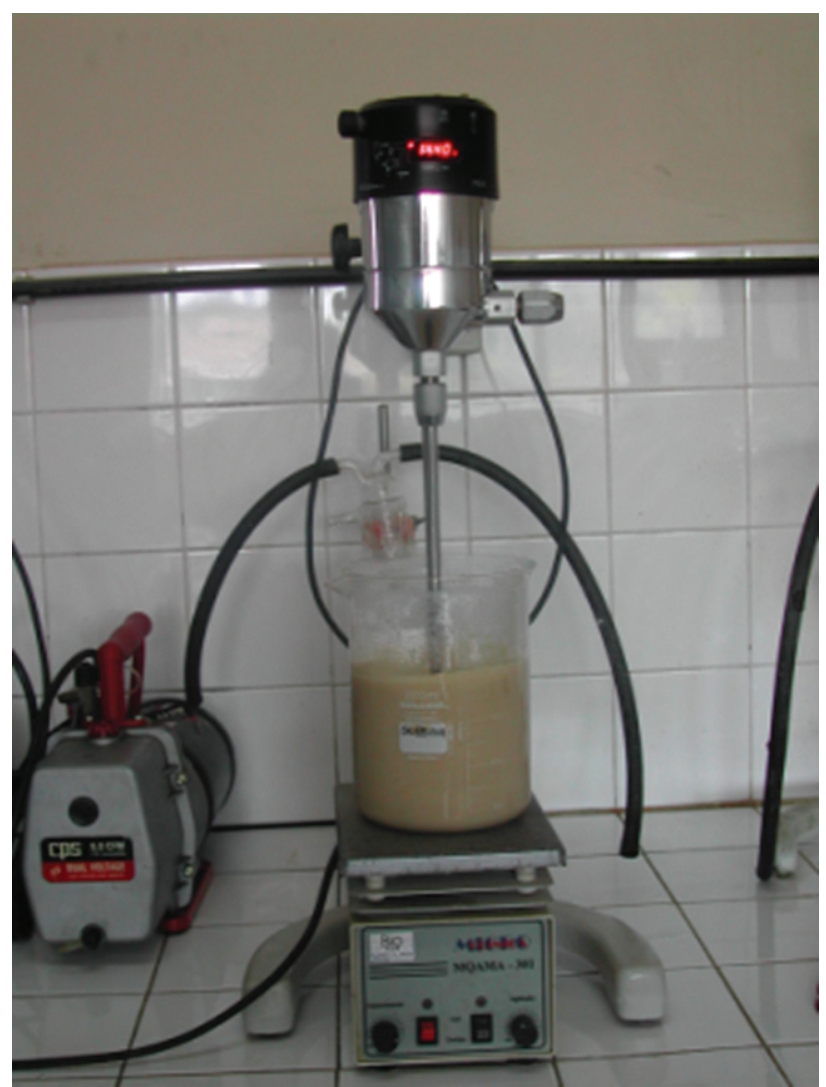

Figura 2. Equipamento para tratamento da argila.

\subsection{Planejamento Fatorial}

Para avaliar a influência das variáveis de entrada (teor de argila e número de camadas "ativas") sobre as propriedades estudadas foi utilizado um planejamento fatorial do tipo $2^{2}$ com três experimentos no ponto central, totalizando sete experimentos.

A matriz de planejamento experimental fatorial utilizada está representada na Tabela I. Os valores codificados e reais das variáveis de entrada, empregados no planejamento encontram-se na Tabela 2.

A regressão dos dados experimentais é realizada utilizando o programa STATISTICA ${ }^{\text {TM }}$ (Statsoft). ${ }^{(14,15)}$

\subsection{Aplicação dos Revestimentos}

As aplicações das películas dos materiais nanoestruturados polímero/argila sobre os corpos de prova metálicos, a partir de chapas de aço 1020, foram realizadas por meio de uma pistola de spray acoplada a um compressor de ar comprimido, com período de intervalo entre a aplicação das camadas sucessivas de 30 minutos, em estufa a $45^{\circ} \mathrm{C}$. Antes da aplicação dos revestimentos, foi realizada uma limpeza nas superfícies dos corpos de prova para remoção da "carepa" de laminação, ferrugem ou vestígios de graxas ou óleos, segundo especificações da norma N-0005 (Petrobras, 2003).(16) Essa limpeza é 
feita por meio de solventes, que, para este trabalho, foi a acetona P.A. $\left(\left(\mathrm{CH}_{3}\right)_{2} \mathrm{CO}\right)$. Após a obtenção das placas já revestidas, estas foram submetidas a ensaios de medição de espessura do revestimento, medidas de aderência e exposição ao meio salino, dentre outros.

\subsection{Medidas de Espessura}

Foram realizadas medidas de espessura de película seca nos revestimentos comerciais, segundo a norma ISO 2808. ${ }^{(17)}$

As medidas de espessura de película seca dos revestimentos acrílicos foram realizadas com paquímetro, tomando como referência uma amostra de substrato sem aplicação de revestimento, sendo o resultado final a média de cinco medições em diferentes pontos da amostra.

\subsection{Medidas de Aderência}

Os ensaios de aderência dos revestimentos foram realizados segundo a norma ASTM D 3359, ${ }^{(18)} \mathrm{em}$ todas as amostras estudadas.

\subsection{Ensaios de Exposição a Atmosferas Corrosivas}

Os ensaios de exposição ao meio salino foram realizados através da imersão das placas já revestidas em uma solução aquosa de $\mathrm{NaCl}$, na concentração de $3 \%$ em volume, no qual permaneceu por 360 horas. Esse ensaio

Tabela I. Matriz de planejamento experimental fatorial do tipo $2^{2}$ com três experimentos no ponto central

\begin{tabular}{ccc}
\hline Experimentos & Teor de argila (T) & $\begin{array}{c}\text { Número de camadas } \\
\text { "ativas" (N) }\end{array}$ \\
\hline 1 & -1 & -1 \\
2 & +1 & -1 \\
3 & -1 & +1 \\
4 & +1 & +1 \\
5 & 0 & 0 \\
6 & 0 & 0 \\
7 & 0 & 0 \\
\hline
\end{tabular}

foi adaptado a partir da norma ASTM D 870. (19) Para tanto, as amostras foram acondicionadas em um recipiente contendo a solução, para observações e análises posteriores, durante todo o ensaio, com as superfícies isentas da ação direta de raios solares. Esta solução foi constantemente oxigenada, assim como foi trocada ao primeiro sinal de mudança na coloração ou turvamento.

\subsection{Análise Visual das Amostras}

Todas as amostras foram fotografadas antes e após o ensaio de imersão em solução aquosa de $\mathrm{NaCl}$, e realizadas inspeções diárias para detecção do aparecimento dos diversos tipos de corrosão, conforme especificações descritas em normas ASTM, ABNT e Petrobras.

\section{RESULTADOS}

Primeiramente, foram realizadas as medidas de película seca das amostras de revestimentos comerciais e dos revestimentos, realizadas segundo a norma ISO 2808.(17) As medidas podem ser observadas na Tabela 3.

Pela análise dos resultados apresentados na Tabela 3, observa-se que as medidas de espessura de película seca apresentadas pelo fabricante são uma média aproximada entre os valores máximos e mínimos medidos experimentalmente.

A Tabela 4 apresenta os valores de espessura de película seca obtida para as amostras de revestimentos acrílicos, determinadas com o uso de um paquímetro.

Uma análise conjunta dos resultados apresentados na Tabela 4 indica que o aumento no número de camadas "ativas" dos revestimentos estudados resulta em aumento dos valores de espessura de película seca. Comparando as amostras I e III com uma camada "ativa", pode-se observar que o aumento no teor de argila proporciona maiores valores de espessura. Comportamento similar é apresentado pelas mesmas amostras com três camadas "ativas".

Tabela 2. Níveis codificados e reais das variáveis de entrada do planejamento experimental $2^{2}$ com três experimentais no ponto central

\begin{tabular}{cccc}
\hline Variáveis de entrada & \multicolumn{1}{c}{ Níveis } \\
\cline { 2 - 4 } & - I & $\mathbf{0}$ \\
\hline Teor de argila (\%) & I & 3 & 5 \\
Número de camadas "ativas" & I & 2 & 3 \\
\hline
\end{tabular}

Tabela 3. Espessura dos revestimentos comerciais estudados

\begin{tabular}{cccc}
\hline \multirow{2}{*}{ Revestimentos comerciais } & & Espessura do filme $(\mu \mathrm{m})$ \\
\cline { 2 - 4 } & Mínima & Máxima & 275 \\
CORRPASSIV 4900 & 195 & 293 & 270 \\
CORRPASSIV 4905 & 186 & 146 \\
CORRPASSIV 4934 & 110 & 110 \\
\hline
\end{tabular}


Tabela 4. Espessura de revestimentos acrílicos estudados

\begin{tabular}{cccc}
\hline Amostras & $\begin{array}{c}\text { Teor de argila } \\
(\%)\end{array}$ & $\begin{array}{c}\text { Número de } \\
\text { camadas } \\
\text { "ativas" }\end{array}$ & $\begin{array}{c}\text { Espessura } \\
(\mu \mathrm{m})\end{array}$ \\
\hline Amostra I & 1 & $\mathrm{I}$ & 132 \\
\hline Amostra II & 3 & 3 & 328 \\
\hline \multirow{2}{*}{ Amostra III } & 5 & 2 & 216 \\
\hline
\end{tabular}

Segundo dados da literatura, o número de camadas de revestimento influencia diretamente em sua resistência à corrosão, uma vez que um dos mecanismos de proteção anticorrosiva apresentado pelos revestimentos orgânicos está baseado em sua ação como barreira mecânica ou iônica. ${ }^{(20)}$ Sendo assim, pode-se esperar que os revestimentos com maiores valores de espessura de película seca possuam melhores características anticorrosivas.

Ao fazer um comparativo entre os dados de espessura dos revestimentos comerciais e os revestimentos acrílicos desenvolvidos, pode-se afirmar que é possível se desenvolver revestimentos acrílicos que apresentem espessuras de filme compatíveis com os revestimentos comerciais que são amplamente utilizados. ${ }^{(21)}$

Segundo Ferreira et al., ${ }^{(22)}$ qualquer que seja a finalidade do revestimento, este deve formar com o substrato um conjunto sólido que se mantenha íntegro por toda vida útil do sistema. Desta forma, a integridade física é fator fundamental e precede qualquer outra forma de caracterização.

Pode-se observar, a partir dos resultados obtidos, que apenas a amostra de revestimento acrílico com $5 \%$ de argila organofílica e com três camadas "ativas" não apresenta sinais de destacamento quando solicitadas por uma tensão adesiva.

Segundo a norma NBR I I003, ${ }^{(20)}$ a aderência dos revestimentos acrílicos, aditivados com teores de I, 3 e $5 \%$ de argila organofílica, varia entre $X_{0} Y_{0}$ e $X_{2} Y_{2}$, isto é, sob a ação de uma força de tração adesiva. Os revestimentos apresentam graus de destacamento de $2 \mathrm{~mm}$ ao longo da direção do corte $\left(X_{2}\right)$ e $4 \mathrm{~mm}$ na intersecção ao corte $\left(\mathrm{Y}_{2}\right)$ a nenhum destacamento $\left(\mathrm{X}_{0}, \mathrm{Y}_{0}\right)$. Portanto, tanto o aumento no teor de argila organofílica, de $1 \%$ a $5 \%$ em peso, como também uma maior espessura do revestimento, resulta na obtenção de melhores resultados de resistência ao destacamento, ou seja, melhor aderência.

A Tabela 5 apresenta os resultados obtidos a partir da análise das amostras com revestimento acrílico, e pode-se observar que, após 360 horas de exposição em ensaio de imersão em solução aquosa a $3 \%$, cada uma das amostras de revestimentos estudadas apresenta sinais distintos de empolamento, segundo a norma ASTM D7I4,(23) e enferrujamento, segundo a norma ASTM D6 I0.(24)
Tabela 5. Grau de enferrujamento e grau de formação de bolhas apresentados pelas amostras de revestimentos acrílicos

\begin{tabular}{ccc}
\hline Experimentos & $\begin{array}{c}\text { Grau de } \\
\text { enferrujamento (\%) }\end{array}$ & $\begin{array}{c}\text { Grau de formação } \\
\text { de bolhas }\end{array}$ \\
\hline 1 & 10 & 4 \\
2 & 16 & 8 \\
3 & 33 & 4 \\
4 & 1 & 0 \\
5 & 10 & 6 \\
6 & 10 & 8 \\
7 & 10 & 6 \\
\hline
\end{tabular}

As amostras sem revestimento apresentam intenso processo corrosivo, quando submetido aos ensaios supracitados.

A partir de uma análise conjunta (ensaios de enferrujamento e empolamento e análise visual) de todas as amostras de revestimento estudadas, pode-se indicar que a adição de crescentes teores de argila organofílica bem como o aumento no número de camadas "ativas" conduz os revestimentos a apresentarem melhores características anticorrosivas.

A Tabela 6 apresenta as análises de variância e os modelos matemáticos codificados (equação de regressão) para as propriedades de grau de enferrujamento e grau de formação de bolhas dos revestimentos acrílicos estudados.

O coeficiente de determinação ou explicação $\left(R^{2}\right)$ quantifica a qualidade do ajustamento, pois fornece uma medida da proporção da variação explicada pela equação de regressão em relação à variação total das respostas, variando de 0 a $100 \%$.

A análise de significância estatística a partir das equações apresentadas evidencia que o grau de empolamento não é influenciado pelas variáveis de entrada, ou seja, a intensidade de formação das bolhas nas superfícies metálicas não é significativamente afetada quando se varia o teor de argila de $3 \%$ a $5 \%$ ou quando se varia o número de camadas "ativas" aplicadas.

Para o grau de enferrujamento, uma análise da equação presente na Tabela 6 mostra que o teor de argila e a combinação dos fatores, teor de argila e número de camadas aplicadas interferem de forma estatisticamente significativa ao nível de $95,0 \%$ de confiança. Ou seja, a porcentagem de superfície corroída diminui quando do aumento nos teores de argila organofílica. Comportamento similar é apresentado quando se aumentam, simultaneamente, o teor de argila e número de camadas "ativas".

De acordo com as características apresentadas da análise estatística na Tabela 6, para o grau de enferrujamento, a maioria dos efeitos é estatisticamente significativa. 
Tabela 6. Análise estatística do grau de enferrujamento e do grau de empolamento (formação de bolhas), apresentados pelas amostras com revestimento acrílico

\begin{tabular}{ccc}
\hline Amostra & \% de variação explicada \\
\cline { 2 - 3 } & Grau de enferrujamento & Grau de empolamento \\
\hline Revestimento acrílico & 97,0 & 83,0 \\
\hline Modelos matemáticos codificados para os revestimentos acrílicos \\
\hline Grau de enferrujamento (GEN) & GEN $=12,85 *-6,5$ *T $+2 \mathrm{~N}-9,5 * T N$ \\
Grau de empolamento (GEM) & GEM $=5,14 *-2 \mathrm{~N}-2 \mathrm{TN}$ \\
\hline
\end{tabular}

*estatisticamente significativa com $95,0 \%$ de confiabilidade.

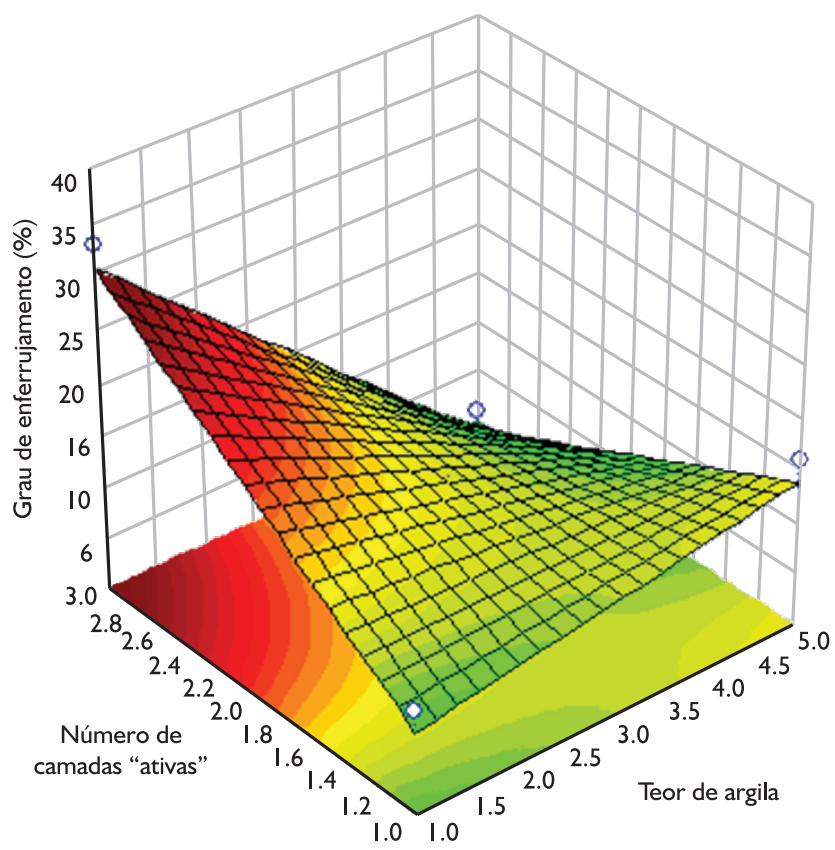

Figura 3. Superfície de resposta do modelo matemático proposto para o grau de enferrujamento.

Como já comentado anteriormente, o mesmo não ocorre para o grau de empolamento, ou seja, neste caso a maioria dos efeitos não é estatisticamente significativa. Este fato é comprovado por meio da análise do modelo codificado apresentado para essa propriedade na Tabela 6 .

A Figura 3 apresenta a superfície de resposta obtida para o modelo matemático proposto para o grau de enferrujamento, presente na Tabela 6.

Pela análise da Figura 3 pode-se observar que o aumento no teor de argila de $1 \%$ a $5 \%$, promove uma melhora no grau de enferrujamento, ou seja, quanto maior é o teor desse sólido na composição do revestimento menor é o enferrujamento observado com o passar do tempo e exposição às intempéries. Observa-se comportamento semelhante ao anterior com o aumento de número de camadas no revestimento, indicando que a composição que apresenta $\circ$ melhor resultado para a propriedade analisada é a amostra do experimento 7 do planejamento experimental, pois, esta apresenta os maiores teores de argila e maior número de camadas "ativas", ou seja, $5 \%$ de argila organofílica e três camadas "ativas".
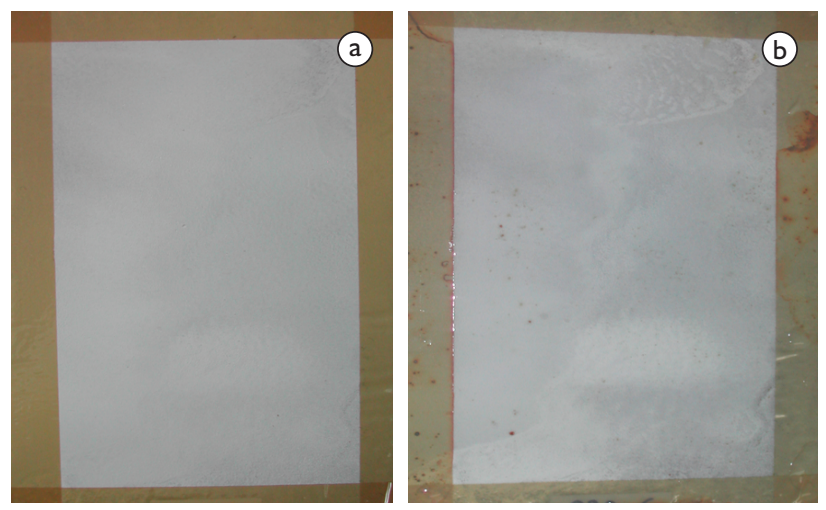

Figura 4. Aspecto visual das amostras com revestimento III: (a) Amostra antes do ensaio; (b) Amostra após exposição de 360 horas na água do mar.

A analise visual das amostras do revestimento acrílico III com três camadas "ativas" (Figura 4) permiti observar que a superfície apresenta pouquíssimos pontos de ferrugem. 
Segundo Martins, ${ }^{(9)}$ denomina-se período de indução o período durante o qual nenhuma degradação é observada. Dentre as amostras estudadas o revestimento III, com três camadas "ativas", é o que apresenta maior período de indução (I 40 horas), ou seja, os primeiros vestígios de ferrugem foram observados no sexto dia de exposição ao meio salino.

Portanto, o revestimento que apresenta os melhores resultados é o revestimento acrílico III e três camadas "ativas". Este, por sua vez, apresenta uma maior espessura da película seca, uma melhor aderência, maior período de indução, e por fim, uma melhor resistência à corrosão, evidenciados pela menor área afetada pela corrosão.

\section{CONCLUSÕES}

Com o objetivo de estudar a influência do teor de argila assim como também do número de camadas ativas dos revestimentos acrílicos, conclui-se:
- O aumento no teor de argila proporciona maiores valores de espessura de película seca; comportamento similar é observado para o aumento do número de camadas "ativas";

- Uma análise estatística dos resultados mostra que o grau de empolamento não é significativamente influenciado pelas variáveis de entrada, teor de argila e número de camadas ativas, ao nível de $95 \%$ de confiança; e

- As melhores características anticorrosivas são observadas para os revestimentos acrílicos aditivados com $5 \%$ de argila organofílica e três camadas "ativas".

\section{Agradecimentos}

As autores agradecem às Empresas Bentonit União Nordeste e Ormecon, pelo fornecimento das argilas comerciais Brasgel PA e dos revestimentos comerciais, respectivamente.

\section{REFERÊNCIAS}

I BERGER, D. M.; SCHWEITZER, P. A. Corrosion and corrosion protection handbook. 2. ed. New York: Marcel Dekker, 1989.

2 SHREIR, L. L.; JARMAN, R. A.; BURSTEIN, G. T. Corrosion: metal/environment reactions. London: ButterworthHeinemann, 2000. p. 38

3 CLINT, J. H. Surfactant aggregation. Glasgow: Blackie, I992. http://dx.doi.org/ I0.1007/978-94-0 I I-2272-6

4 GENTIL, V. Corrosão. 3. ed. Rio de Janeiro: LTC, 1996.

5 EL-ACHOURI, M. et al. Synthesis of some cationic gemini surfactants and their inhibitive effect on iron corrosion in hydrochloric acid medium. Corrosion Science, v. 43, n. I, p. 19-35, May 200 I. http://dx.doi.org/10.10 I6/S00 I0938X(00)00063-9

6 ALSABAGH, A. M.; MIGAHED, M. A.; HAYAM, S. A. Reactivity of polyester aliphatic amine surfactants as corrosion inhibitors for carbon steel in formation water (deep well water). Corrosion Science, v. 48, n. 4, p. 8I 3-828, May 2006.

7 GHASEMI, Z.; TIZPAR, A. The inhibition effect of some amino acids towards Pb-Sb-Se-As alloy corrosion in sulfuric acid solution. Applied Surface Science, v. 252, p. 3667-3672, June 2006. http://dx.doi.org/ I0.10 I6/j. apsusc. 2005.05 .043

8 ABDEL HAMID, Z.; SOROR, T. Y.; EL-DAHAN, H. A. Anti-corrosion ability of surfactants: a review. Anticorrosion Methods \& Mater, v. 45, n. 6, p. 1927-1948, June 1998.

9 MARTINS, P. R. A. Estudo e avaliação de desempenho de polímeros condutores com óxidos de ferro. 1999 . 122 p. Dissertação (Mestrado em Engenharia Química) - Instituto Alberto Luiz Coimbra de Pós-Graduação e Pesquisa de Engenharia - COPPE, Universidade Federal do Rio de Janeiro, Rio de Janeiro, 1999.

I0 WANG, K. H. et al. Synthesis and characterization os maleated polyethylene/clay nanocomposites. Polymer, v. 42 , n. I, p. 9819-9826, Nov. 2007.

I I NAM, H. P. et al. A hierarchical structure and properties of intercalated polypropylene/clay nanocomposites. Polymer, v. 42, n. 23, p. 9633-9640, Nov. 200 I. http://dx.doi.org/l0.10I6/S0032-386I(0I)005I2-2

I 2 GARCÍA LÓPEZ, D.; PICAZO, O.; MERINO, J. C. Polypropylene-clay nanocomposites: effects of compatibilizing agents on clay dispersion. European Polymer Journal, v. 39, n. 5, p. 945-950, May 200 I. http://dx.doi.org/I0.10 I6/ SOOI4-3057(02)00333-6

I 3 RECKNERS, U.; KALNINS, M. Evaluation of the properties of organic coatings by using tape and blistering tests, progress in organic coatings. Progress in Organic Coatings, v. 38, n. I, p. 35-42, Feb. 2000. http://dx.doi.org/I0.1016/ S0300-9440(99)00082-X

I4 BARROS NETO, B.; SCARMINIO, I. S.; BRUNS, R. E. Planejamento e otimização de experimentos. Campinas: Editora da Unicamp, 1996. 
I5 STATSOFT INC. Statistica for Windows, version 5.0. Tulsa, 2000. I CD.

16 PETROBRAS. N-0005: Limpeza de superfícies de aço por ação físico-química. Rio de Janeiro, 2003.

I7 INTERNATIONAL ORGANIZATION OF STANDARDIZATION. ISO-2808 - Paints and varnishes determination of film thickness. Geneva, 2007.

I8 AMERICAN SOCIETY FOR TESTING AND MATERIALS. ASTM D 3359: Standard test methods for measuring adhesion by tape test. West Conshohocken, 1993.

19 AMERICAN SOCIETY FOR TESTING AND MATERIALS. ASTM D870: Standard practice for testing water resistance of coatings using water immersion. West Conshohocken, 2009.

20 ASSOCIAÇÃO BRASILEIRA DE NORMAS TÉCNICAS. Norma NBR I l003: Tintas: determinação da aderência. Rio de Janeiro, 2009.

2I ASSOCIAÇÃO BRASILEIRA DE CORROSÃO - ABRACO. Disponível em: <http://www.abraco.org.br>. Acesso em: 10 set. 2010.

22 FERREIRA, M.; CAMARGO JUNIOR, S. S.; SILVA, R. L. G. Recobrimento interno de dutos para minimização de depósito, fricção e corrosão. In: SEMINÁRIO BRASILEIRO DE DUTOS, 2., 1999, Rio de Janeiro. Anais... Rio de Janeiro: Petrobras, 1999.

23 AMERICAN SOCIETY FOR TESTING AND MATERIALS. ASTM D7 I4: Standard test method for evaluating degree of blistering of paints. West Conshohocken, 2009.

24 AMERICAN SOCIETY FOR TESTING AND MATERIALS. ASTM D 610: Standard practice for evaluating degree of rusting on painted steel surfaces. West Conshohocken, 1985.

Recebido em: 02/07/2012

Aceito em: 05/10/2012 